\title{
CORONAVIRUS AND MULTIPLE SCLEROSIS: RESULTS OF A CASE/CONTROL
}

\section{LONGITUDINAL SEROLOGICAL STUDY}

\author{
C.M. Johnson-Lussenburg and $Q$. Zheng \\ Deparment of Microbiology and Immunology \\ School of Medicine, University of ottawa \\ ottawa, Ontario, CANADA. KIM 8M5
}

\section{INTRODUCTION}

Epidemiological and some clinical evidence has supported a viral hypothesis in the etiology of MS (Johnson, 1975; Waksman, 1983). In addition to rabies, herpes simplex, measles and canine distemper viruses, human coronavirus is one of the candidates. However, so far, a viral cause for MS remains only an attractive hypothesis not an established fact and none of the proposed candidate viruses have been shown to be unequivocally associated with the cause of MS.

With respect to coronaviruses, the difficulty of direct association by isolation is well recognized because they are notoriously difficult to isolate (Burks et al, 1981). And, although coronavirus particles have been observed in tissues from MS patients (Tanaka et al, 1976), the implication of a coronaviral agent in the etiology of MS has been attempted mostly by indirect methods, namely serology, with equivocal but mainly negative results (Hovanec and Flanagan, 1983; Madden et al, 1981; Salmi et al, 1982). In these earlier studies however, the prevalence of coronavirus infections in the communities surveyed was not documented and no information about seasonal factors was provided. The latter is considered important because of the seasonal incidence of coronaviral infections (Cavallero and Monto, 1970; McIntosh et al, 1970). Thus, since these sera were random, individual samples and not paired or sequential repeats, it was not possible to distinguish acute coronavirus infections from the putative persistent viral involvement in MS, both of which could be reflected by elevated antibody titres.

The present investigation was designed to seek serological evidence of a role for coronavirus in MS by comparing serum antibody titres among the same patients and their respective controls over an extended period of time. Since human coronavirus causes the 'common cold', in order to cover the putative 'cold season' (late winter and early spring), a minimum period of 8 months was considered essential. The case/control study started in october 1982 and ended in May, 1983. The aim of this study was to try to demonstrate a difference in the immune response of persons with MS compared to their controls as reflected by the levels of circulating HCV/229E antibody correlated with the occurrence of upper respiratory infections (both groups) and/or exacerbations of MS symptoms (patients). 
Table 1. Comparison of MS case and control subjects.

\begin{tabular}{|c|c|c|}
\hline & Cases & Controls \\
\hline Number & 39 & 39 \\
\hline $\begin{aligned} \text { Controls: } & \text { Spouse } \\
& \text { Non-spouse }\end{aligned}$ & $\begin{array}{l}20 \\
19\end{array}$ & $\begin{array}{l}20 \\
19\end{array}$ \\
\hline $\begin{aligned} \text { Sex: } & \text { male } \\
& \text { female }\end{aligned}$ & $\begin{array}{l}10(25.68) \\
29(74.48)\end{array}$ & $\begin{array}{l}15(38.58) \\
24(61.58)\end{array}$ \\
\hline Average age & 38.8 years & 39.4 years \\
\hline siblings & 3.15 & 3.26 \\
\hline Family history of MS & 20.58 & $2.68 \quad(P<0.01)$ \\
\hline Urban Inhabitant & $28(71.88)$ & $23(60.58)$ \\
\hline \multicolumn{3}{|l|}{ Family pets: } \\
\hline $\begin{array}{l}\text { Dogs } \\
\text { Cats }\end{array}$ & $\begin{array}{l}598 \\
568\end{array}$ & $\begin{array}{l}588 \\
508\end{array}$ \\
\hline
\end{tabular}

\section{MATERIALS AND METHODS}

Subjects. Through the Multiple sclerosis clinic at the ottawa General Hospital, Ottawa, Canada, and with the collaboration of its Director, Dr. R.F. Nelson, area MS patients were invited to participate in the study. They were requested to provide their own 'pal' controls; either a spouse, relative or close associate (neighbour, friend or colleague at work) who would be willing to participate for the duration of the 8 month study. These controls were defined and selected according to criteria necessary for statistical evaluation (C.I. Neutel, personal communication). Thirtynine MS patients and appropriate controls volunteered giving a total of 78 individuals which could be further sub-divided, if necessary, on the basis of control types i.e. spouse controls (20) who were age and environmentally matched versus non-spouse controls (19) who were age and sex-matched and had a common community environment.

The characteristics of both groups are summarized in Table 1 . The information on which this comparison is based was obtained from two questionnaires, one given at the beginning and the other at the conclusion of the study. It can be seen that, except for MS, both groups were remarkably similar indicating that the control group was appropriate to the study. In the second questionnaire, an attempt was made to document information believed at one time to be relevant to the development of MS; namely whether, during childhood and up to age 15, the participants had lived in a mainly rural or urban environment, had prolonged, close contact with animals such as family pets or farm animals or knew of schoolmates who subsequently developed MS. As can be seen, there was no difference between the cases and the controls. However, there was a highly significant difference between the groups with respect to a family history of MS ( $P$ (0.01). This finding is in agreement with other reports of a genetic predisposition to the disease (Kurtzke, 1980; Waksman, 1983).

Serum specimens were obtained from each MS patient and their control at monthly intervals according to a fixed schedule over the 8 month study period. All test sera were coded by sequential numbering of randomly selected specimens and the code was not broken until all testing was completed. Of a possible 624 sera from all participants, 584 were obtained giving a 948 compliance rate. All sera were heat-inactivated and 
In addition to the case/contr: 325 Red cross blood donors, in batches of 25, were collected at weekly intervals over a 13 week period from March to June, 1983.

Serological Assay. The indirect immunofluorescence assay (IFA) was used for this study (Pedersen et al. 1978). Ll32 cells persistently infected with HCV/229E, termed HV cells (Chaloner-Larsson and Johnson-Lussenburg, 1981), were grown as monolayers in 8-well slide chambers (LabTek, Miles Scientific), fixed in acetone and stored at $-20^{\circ} \mathrm{C}$ until required. Sera were first screened at dilutions of $1: 20$ and all positive reactions were titrated $(1: 20$ through $1: 160)$. Controls were included with each test series and consisted of homologous and heterologous positive standards (absorbed guinea pig anti-HCV/229E hyperimmune and convalescent antiFeline Infectious peritonitis (FIP) sera respectively) and negative sera and/or phosphate buffered saline. Fluorescein conjugates were obtained commercially (zymed Laboratories, San Francisco, CA) and used at recommended dilutions. Slides were examined, by the same individual, for specific immunofluorescence using a Leitz Laborlux II epi-fluorescence microscope.

The distinctive and characteristic pattern of positive, fluorescing cells in the persistently infected monolayer as detected by the control absorbed guinea pig anti-229E serum is shown in Fig. 1A. This pattern of single or dividing fluorescing cells was easily distinguished from nonspecific reactions which were sometimes encountered with the test sera. A further positive control for antigen specificity was provided by the cross-reaction between $229 \mathrm{E}$ antigen in the persistently infected and the convalescent FIP antisera (Fig. 1B). These control reactions provided continuing support for the suitability of the persistently infected cells as the basis for our serological assay.

Statistical Methods. Statistical analyses were carried out in collaboration with Dr.J. Last and Dr. S.Narod of the Department of Epidemiology and Community Medicine, University of Ottawa. The MS and control groups were sub-divided on the basis of their history of common cold (upper respiratory) infections and other information provided by both questionnaires. Records of the occurrence of respiratory infections and/or MS exacerbations were obtained monthly throughout the study. Exacerbations were
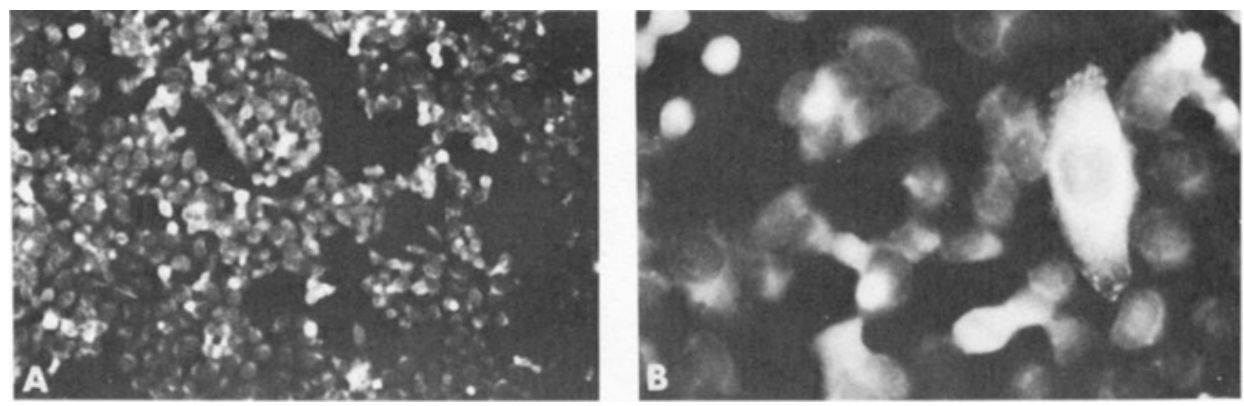

Fig. 1. A. The characteristic and distinctive pattern of immunofluorescing cells in persistently infected HV monolayer detected by host absorbed anti-229E hyperimmune guinea pig serum. Note single or double fluorescing cells. B. Specific cross reaction between $229 \mathrm{E}$ antigen-containing HV cells and convalescent FIP serum used to confirm specificity of antigen in the monolayer. 

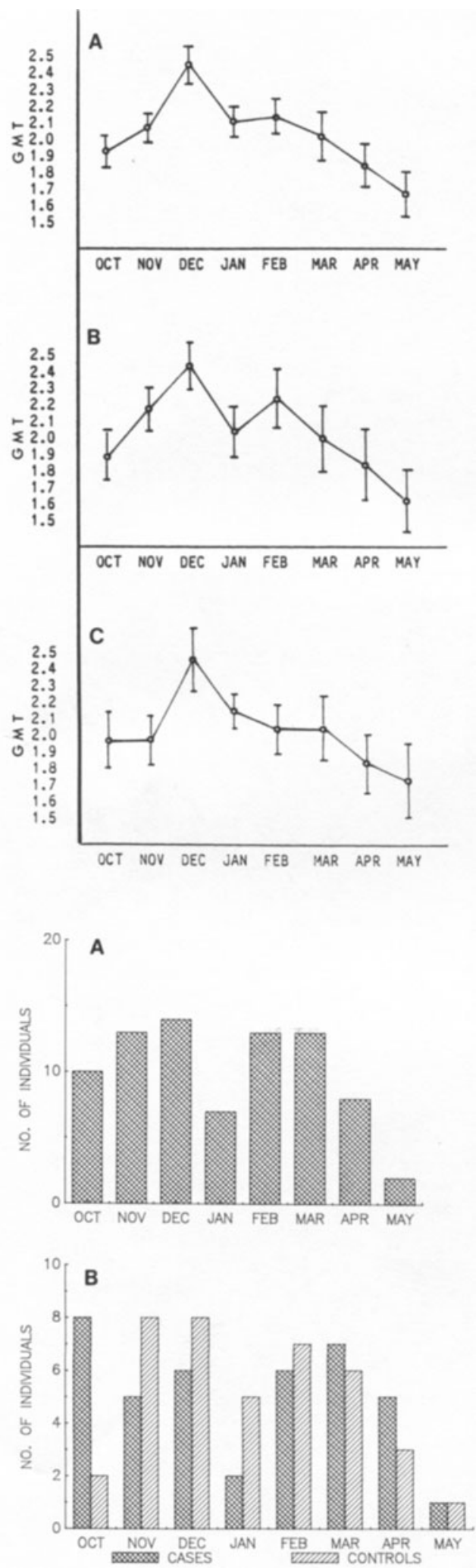

Fig. 2. Monthly average geometric mean titres (GMT) of sera from MS patients and their controls from October 1982 to May 1983.
A. All participants.
B. MS patients.
C. Controls.

Fig. 3. Number of colds reported by month.
A. All participants.
B. Comparison of MS patients and their controls.


Table 2. Comparison of geometric means of average IFA titers of MS cases and controls.

\begin{tabular}{lcc}
\hline & Average titer & P value \\
\hline Case & $1.957 \pm 0.109$ & 0.90 \\
Control & $1.950 \pm 0.127$ & \\
Cold history* & & \\
$\quad$ No cold & $1.830 \pm 0.66$ & \\
One cold & $1.890 \pm 0.107$ & 0.30 \\
Two colds & $2.250 \pm 0.227$ & \\
Three colds & $1.966 \pm 0.203$ & \\
Age** & & \\
< 44 & $1.940 \pm 0.113$ & 0.90 \\
$>44$ & $1.944 \pm 0.123$ & \\
Sex & & \\
Male & $1.984 \pm 0.153$ & 0.80 \\
Female & $1.939 \pm 0.100$ & \\
\hline
\end{tabular}

* Based on the information provided in the final questionnaire. The participants were divided into groups according to their reported frequencies of colds experienced during the 12 months prior to the study.

** Age at which individuals segregated equally for statistical testing.

confirmed through the MS Clinic and details of URIs were obtained by personal reporting. Comparisons between cases and controls were done using conventional unpaired statistical analysis. Paired analyses were used in comparing monthly changes in antibody titre. Coding of serum titres using logarithmic transformation and calculation of the geometric mean titres (GMT) were performed according to white (1973).

Computer analyses were performed using BMDP statistical software (7D) for the selected tests.

\section{RESULTS AND DISCUSSION}

Analysis of Immunofluorescence Assay Results. The IFA titres for the 584 MS/pal control sera are shown in Table 2. The GMT for these two groups

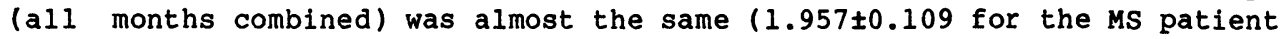
group and $1.950 \pm 0.127$ for the control group). The $P$ value of 0.90 indicated no statistically significant difference. Further analysis of the data obtained from the final questionnaice showed that the GMT was also not affected by the frequency of colds, age, or sex. These results, therefore, suggest that there was no major difference in the frequency of colds reported by all the individuals in this study.

The monthly comparison of the GMT between the MS case/control groups is shown in Fig. 2. The antibody levels for both groups peaked in December (GMT of 2.42 for MS cases and 2.45 for the controls) then gradually decreased, but there were no significant differences in the seasonal patterns of these two groups.

As seen in Fig. 3, the analysis of the relationship between the GMT of MS patients and controls based on the colds which they experienced during the 8 month study period also showed no significant differences. The total 
Table 3. Relationship between Colds and GMT of MS cases and controls.

Average GMT

\begin{tabular}{lll} 
All months & $2.046 \pm 0.064$ & \\
'Cold' months* & $2.114 \pm 0.115$ & 2.00 \\
'No cold' months & $2.028 \pm 0.068$ & 2.05 \\
& & 2.28 \\
Month prior to cold & $2.020 \pm 0.109$ & \\
Month of cold & $2.114 \pm 0.115$ & \\
Month after cold & $2.070 \pm 0.122$ & \\
\hline
\end{tabular}

number of colds reported by both groups was 40 . The lack of significant correlation between the average titer and frequency of cold by month was indicated by Pearson's correlation coefficient of 0.733 . The data in Table 3 showed that the average titres did not change with the occurrence of colds. The average titre for all months was $2.046 \pm 0.064 ; 2.114 \pm 0.115$ for 'cold' months and $2.028 \pm 0.068$ for 'no cold' months. A 'cold' month was defined as that month in which an individual experienced a cold versus a 'no cold' month when no cold was reported. To further test for an association, we examined MS patients' GMTs grouped according to the months before and after as well as during the 'cold' month. As can be seen in Table 3, the average titres of these three groups were not significantly different. In summary, therefore, these results indicate that there was no group correlation of cold symptoms to titers in either the MS cases or between the cases and controls.

Association of Colds with Exacerbations of MS. We next tested for a correlation between the occurrence of colds and MS exacerbations within the patient group. In the total 294 months of case observation, the monthly cold frequency was 13.38, almost identical to that of 13.78 seen in the control population ( 40 colds/293 months). There were 19 exacerbations of MS and 39 episodes of colds. Of the 19 MS exacerbations, 6 occurred in association with colds and 13 did not while in the remaining 275 nonexacerbation months, 33 were associated with colds and 242 were not. The rate of exacerbation was 3.38 times higher in 'cold' months than in the 'no cold' months ( $p=0.0284$, Fisher's exact test, one-tailed) and was considered significant (Table 4 ).

Table 4. Relationship of MS Exacerbations and Colds.

\begin{tabular}{|c|c|c|c|}
\hline & & $1 d$ & \\
\hline & Yes & No & \\
\hline Yes & 6 & 13 & 19 \\
\hline Exacerbation No & 33 & 242 & 275 \\
\hline & 39 & 255 & 294 \\
\hline & & & $P<0.05$ \\
\hline
\end{tabular}


These ratios are similar to those obtained by sibley et al. (1985) who reported that 158 of infections were associated with worsening of MS whereas 328 of MS exacerbations coincided with colds during the same period. Although colds were most frequently reported during the winter months, they reported little seasonality evident in the MS episodes.

Association of HCV/229E colds with MS cases and controls. For further analysis of the colds experienced by the MS patients and controls it was possible to correlate colds with four-fold or higher serological rises using the BMDP statistical program. Since these peaks reflected the presence of $\mathrm{HCV} / 229 \mathrm{E}$ antibodies, we could determine the number of $\mathrm{HCV} / 229 \mathrm{E}$ colds within each group. These results are summarized in Fig.4. of the total 80 colds ( 40 in each group), 46 (57.58) were identified as being associated with HCV/229E; 28 in the cases group and 18 in the controls. statistical analysis showed that there were significant differences between these groups ( $P<0.05$ ) and we concluded that there was a positive association of coronaviral 229E infection with MS (Table 5).

Presence of $\mathrm{HCV} / 229 \mathrm{E}$ in the General Community. Although the total period of time covered by this study was too short to draw any firm conclusions, the results of our tests on the "community control" sera indicated an increase in coronavirus activity in the late spring/early summer of 1983, from an average positive rate of 408 to 52,64 and 728 in early June. These results clearly indicated the presence of $\mathrm{HCV} / 229 \mathrm{E}$ in the general community with an increase in coronavirus activity in the late spring/ early summer of 1983. However, it was not possible to demonstrate seasonal incidence; neither was it possible to correlate the presence of anti-
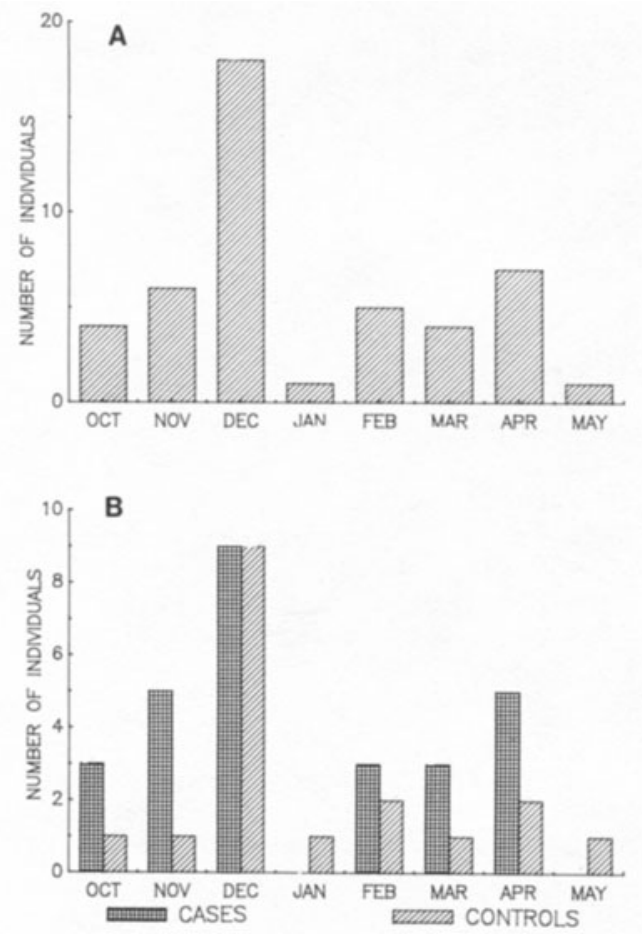

Fig. 4. Number of $\mathrm{HCV} / 229 \mathrm{E}$ colds identified by a four-fold rise in antibody titre.

A. All participants.

B. Comparison of MS patients and their controls. 
Table 5. Comparison of HCV/229E colds among MS cases and controls.

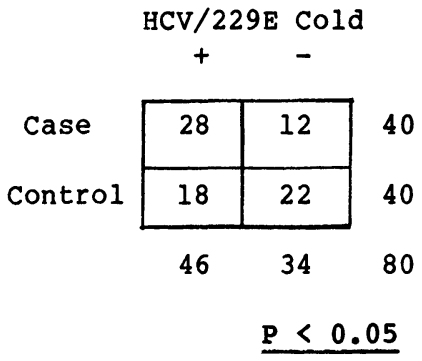

bodies with clinical respiratory disease. Unfortunately, these results also did not provide the background information on the incidence of $\mathrm{HCV} / 229 \mathrm{E}$ in the community which might have been relevant to the MS case/ control study. We do not fully understand why both the cases and controls showed such a high rate of positivity (95-988 during any study month) but speculate that there was better opportunity for common exposures to and transmission between both the spouse and non-spouse control participants.

\section{CONCLUDING REMARKS}

The aim of this study was to try to provide a link between MS and human coronavirus by demonstrating a difference in the immune response of persons with MS compared to their controls as reflected by the levels of circulating HCV/229E antibody. Our results have shown no statistically significant differences between in the serological reactions of MS patients and controls with respect to the persistent $229 \mathrm{E}$-derived VH virus in the IFA. Several possible reasons for this negative finding might be suggested.

First and foremost, the sample size was too small to resolve potential differences between the patients and their controls. This is particularly the case with such a high level of positive reactors in both groups and the circulation of coronaviruses in the community. If there was indeed any difference to be detected by serology, far greater numbers would be required to compensate for normal serum fluctuations in addition to responses to repeated virus exposures.

It is possible that our MS patients were, in fact, "over-controlled". To determine whether there was any bias between the spouse and non-spouse control groups $(20$ and 19 pairs respectively) which might reflect trasmission of viral infections due to closer contact between spouses, we classified the subjects accordingly and again tested for coronavirus:cold association. No significant differences were found in the numbers of coronaviral infections in either of these groups, confirming that the controls, both spouse and non-spouse, were reliable. However, if virus was being maintained within this selected population, again, the sample size was too small to resolve potential differences. Furthermore, in this case, the duration of the study was also too short.

Thus, it might be concluded that there was no association between MS and coronavirus 229E. However, we consider that, to date, the results are not clear cut enough to rule out coronaviruses. The two findings which support this opinion are the significant association of colds with exacerbation of MS and the equally significant association of coronavirus 229E infection with MS patients in comparison to controls. Although these latter findings are not strong evidence of a definite, etiological link 
between coronavirus and MS, they do provide tenuous support for a positive association which requires further elucidation.

\section{ACKNOWLEDGEMENTS}

We are especially grateful to all the MS patients and their controls without whose participation and commitment this study would not have been possible. We also thank Donelda Taylor and Lise Ray for their excellent technical assistance and the ottawa General Hospital for permitting us to use their facilities.

This work was supported by grants from the Multiple sclerosis society of Canada and the International Development Research Centre. Qz was the holder of an IDRC Scholarship.

\section{REFERENCES}

Burks, J.S., Devald, B.L., Janovsky, L.D. and Gerdes, J.C. (1980). Two coronavirues isolated from central nervous system tissue of two multiple sclerosis patients. Science 209: 933-934.

Cavallaro, J.J. and Monto, A.S. (1970). Community-wide outbreak of infection with 229E-like coronavirus in Tecumseh, Michigan. J. Inf. Dis. 122: 272-279.

Chaloner-Larsson, G. and Johnson-Lussenburg, C.M. (1980). Establishment and maintenance of a persistent infection of $L 132$ cells by human coronavirus, strain 229E. Arch. Virol. 69: 117-129.

Hovanec, D.L. and Flanagan, T.D. (1983). Detection of antibodies to human coronaviruses $229 \mathrm{E}$ and $0 \mathrm{C} 43$ in the sera of multiple sclerosis patients and normal subjects. Infec. Immun. 41: 426-429.

Johnson, R.T. (1975). The possible viral etiology of multiple sclerosis. Adv. Neurol. 13: 1-46.

Kurtzke, J.F. (1980). Epidemiology contributions to multiple sclerosis: A review. Neurology 30: 61-79.

Madden, D.L., wallen, W.C., Houff, S.A., Leinikki, P.A., Sever, L., Holmes, K.A., Castellano, G.A. and Shekarchi, I.C. (1981b). Coronavirus antibodies in sera from patients with multiple sclerosis and matched controls. Arch. Neurol. 38: 209-210.

McIntosh, K., Kapikian, A.Z., Turner, H.C., Hartley, J.W., Parrott, R.M. and Chanock, R.M. (1970). Seroepidemiologic studies of coronavirus infection in adults and children. Am. J. Epidemiol. 91: 585-592.

Pedersen, N.C., Ward, J., and Mengeling, W.L. (1978). Antigenic relationship of the feline infectious peritonitis virus to coronaviruses of other species. Arch. Virol. 58: 45-53.

Salmi, A., Ziola, B., Hovi, T. and Reunanen, M. (1982). Antibodies to coronaviruses $0 \mathrm{C} 43$ and $229 \mathrm{E}$ in multiple sclerosis patients. Neurol. 32: 292-295.

Sibley, W.A., Bamford, C.R., and Clark, K. (1985). Clinical viral infections and multiple sclerosis. The Lancet I: 1313-1315.

Tanaka, R., Iwasaki, Y. and Koprowski, H. (1976). Intracisternal viruslike particles in brain of multiple sclerosis patients. J. Neurol. sci. 28: 121-126.

Waksman, B.H. (1983). Viruses and immune events in the pathogenesis of multiple sclerosis. In: Viruses and demyelinating diseases, edited by C.A. Mims, M.L. Cuzner and R.E. Kelly. pp 151-165. Academic Press, N.Y.

White, C. (1973). Statistical methods in serum surveys. In: serological Epidemiology, edited by J.R. Paul and C. White, pp. $\overline{19}-32$. Academic Press, New York and London. 